\title{
TESTES DE CAMPO COMPARATIVOS ENTRE FONTES E GEOFONES VISANDO À INVESTIGAÇÃO SÍSMICA RASA EM ÁREAS URBANAS
}

Oleg Bhokonok*, Renato Luiz Prado**, Liliana Alcazar Diogo**

*IAG/USP - Programa de Pós-graduação

${ }^{* *}$ IAG/USP - Departamento de Geofísica

Copyright 2004, SBGf - Sociedade Brasileira de Geofísica

Este texto foi preparado para a apresentação no I Simpósio de Geofísica da Sociedade Brasileira de Geofísica, São Paulo, 26-28 de setembro de 2004. Seu conteúdo foi revisado pela Comissão Tecno-científica do I SR-SBGf mas não neceudo iomente representa a opinião da SBGf ou de necessariami revisado pela Comissão Tecno-cientifica do $I$ SR-SBGf mas na a reprodução total ou parcial deste material para propósitos comerciais sem prévia autorização da SBGf.

\section{Resumo}

Visando avaliar a potencialidade do uso da sísmica rasa de reflexão na investigação geológica - geotécnica rasa, em ambientes urbanos ruidosos e com superfície pavimentada e também a fim de entender melhor a importância da escolha e do modo de uso das ferramentas disponíveis foram efetuados diversos ensaios de pseudo-análise de ruído. $\mathrm{Na}$ aquisição dos dados foram empregados um sismógrafo de 24 canais, geofones de 28 e $100 \mathrm{~Hz}$ e como fonte geradora utilizouse o impacto de uma marreta sobre base metálica e o disparo de um rifle sísmico de calibre 12" em furos de aproximadamente $0,4 \mathrm{~m}$. Melhores resultados foram obtidos com: (i) geofones de $100 \mathrm{~Hz}$ com ponteira de $0,18 \mathrm{~m}$ e fonte marreta com placa de metal; (ii) geofones de $100 \mathrm{~Hz}$ acoplados através de argila na cobertura asfáltica com fonte marreta aplicada sobre asfalto.

\section{Introdução}

A partir dos anos 90 verificou-se na cidade de São Paulo um aumento das obras civis voltadas às necessidades de infra-estrutura e, seguindo uma tendência mundial, elas têm se concentrado no espaço subterrâneo (instalações de túneis viários, reservatórios de retenção de cheias e equipamentos como cabos elétricos e de fibra ótica, etc.). Para a elaboração dos projetos construtivos e de execução dessas obras é necessário um bom conhecimento geológico tais como, distribuição areal e das espessuras dos estratos geológicos, reconhecimento e posicionamento das estruturas geológicas.

A sísmica de reflexão rasa apresenta, portanto, um grande potencial de aplicação uma vez que permite mapear as estruturas de maneira contínua, interpolando de maneira segura as informações dos furos de sondagem (Hunter et al., 1984; Jeng, Y., 1995; Steeples, D.W., and Miller, R.D., 1990).

O meio urbano apresenta, contudo, diversos aspectos que prejudicam ou dificultam a aquisição e análise dos dados sísmicos, como: espaço de operação restrito e estratos superficiais alterados (aterros e cobertura asfáltica), além de ruídos de fundo associados às linhas de alta tensão e ruído vibracional do tráfego.

Esses fatores têm contribuído para que a sísmica de reflexão ainda não seja considerada no Brasil um método investigativo eficaz para os propósitos dos construtores de grandes obras civis urbanas (Prado et al., 2001).

Visando investigar a acurácia e potencialidade do método de sísmica de reflexão rasa nas condições acima descritas é que estão sendo realizados diversos ensaios de campo que compõem o projeto de mestrado de um dos autores.

Aqui são apresentados alguns resultados parciais de ensaios de campo com diferentes tipos de geofones e fontes e condições de acoplamento em uma área de estudo na área urbana da cidade de São Paulo.

São apresentados e discutidos resultados preliminares de testes comparativos entre geofones de diferentes freqüências e fixados através de ponteiras e de argila, em solos e sobre a cobertura asfáltica, bem como resultados de testes comparativos entre as fontes sísmicas do tipo marreta e rifle sísmico.

\section{Metodologia/ Problema Investigado}

Foram executados vários testes de pseudo-análise de ruído, ou seja, enquanto o arranjo de geofones mantevese fixo, variou-se a posição da fonte em intervalos regulares. Uma parte dos testes foi efetuada sobre a cobertura asfáltica e outra diretamente no solo, utilizando diferentes parâmetros de aquisição. (Tabela 1)

A escolha do local desses testes foi condicionada por diversos fatores: i) existência de sondagens mecânicas nas proximidades do local; ii) possibilidade de fazer a aquisição simultânea sobre a cobertura asfáltica e solo; iii) estar situada em local típicamente urbano, com intenso tráfego de veículos e pessoas.

A geologia da área consiste de sedimentos terciários da Formação Itaquaquecetuba da Bacia Sedimentar de São Paulo que assenta-se sobre o embasamento précambriano. A descrição do poço produtor PP1 situado próximo ao local dos ensaios traz a seguinte informação sobre as principais litofácies do local: (1) areia até a profundidade de $25 \mathrm{~m}$; (2) conglomerado de $25 \mathrm{~m}$ a $35 \mathrm{~m}$; (3) areia de $35 \mathrm{~m}$ a $52 \mathrm{~m}$; (4) argila de $52 \mathrm{~m}$ a $70 \mathrm{~m}$; (5) granito-gnaisse do embasamento.

Os ensaios foram realizados empregando geofones de freqüência natural de 28 e $100 \mathrm{~Hz}$, acoplados ao solo com ponteiras de $0,1 \mathrm{~m}$ e $0,18 \mathrm{~m}$ de comprimento e fixados com argila na área pavimentada. No solo foram utilizados dois tipos de fontes: (1) rifle sísmico de calibre 12 , detonados em furos de aproximadamente $0.4 \mathrm{~m}$; (2) marreta de $6 \mathrm{~kg}$ impactada sobre placa de ferro. Sobre o pavimento foi utilizada somente a marreta, com impactos sobre a placa e diretamente sobre o asfalto.

A partir da análise dos dados das sondagens mecânicas, foram realizados alguns testes de modelagem usando o algoritimo Triseis do aplicativo Seismic Unix (Stockwell \& Cohen, 1998) para estimar os possíveis tempos de 
trajetória das ondas refletidas nas interfaces presentes (Figura 1). Concluiu-se que os tempos de chegadas das ondas refletidas estariam dentro de uma janela temporal de no máximo $100 \mathrm{~ms}$, daí a escolha da janela de registro de 200 ms (Tabela 1).

O objetivo principal desses ensaios de campo foi comparar as respostas dos diferentes geofones, em situações distintas de acoplamento e com a geração das ondas por diferentes fontes sísmicas, e a partir desse estudo procurar estabelecer os equipamentos e parâmetros ideais para melhorar a eficácia e resolução do métoda da sísmica de reflexão rasa.

O estudo comparativo foi feito a partir da análise dos espectros de amplitude obtidos nas diferentes condições do ensaio, bem como pelo resultado final do processamento dos sismogramas.

Tabela 1: Parâmetros de aquisição

\begin{tabular}{|l|c|}
\hline Número de canais & 24 \\
\hline Offset mínimo & $0,5 \mathrm{~m}$ \\
\hline Offset máximo & $72 \mathrm{~m}$ \\
\hline $\begin{array}{l}\text { Espaçamento entre } \\
\text { geofones }\end{array}$ & 0,5 \\
\hline Geofones & $28 \mathrm{~Hz}$ e $100 \mathrm{~Hz}$ \\
\hline Fontes & $\begin{array}{c}\text { Rifle (12') e marreta de } \\
6 \mathrm{Kg}\end{array}$ \\
\hline Acoplamento geofones & $\begin{array}{c}\text { Ponteira (0,1 m e 0,18 } \\
\mathrm{m}), \text { argila }\end{array}$ \\
\hline Intervalo de amostragem & $0,25 \mathrm{~ms}$ \\
\hline Comprimento do registro & $200 \mathrm{~ms}$ \\
\hline $\begin{array}{l}\text { Filtro corta-baixas } \\
\text { analógico }\end{array}$ & $3 \mathrm{~Hz}$ \\
\hline
\end{tabular}

Finalmente, numa segunda etapa de testes de campo, após a interpretação dos sismogramas obtidos anteriormente efetuou-se novo teste de ruído com 5 arranjos lineares equidistantes de $0,4 \mathrm{~m}$ e com 4 geofones cada um, equiespaçados de $1 \mathrm{~m}$. Desta forma, e com offsets de 20, 40 e 60 metros, foram adquiridos novos registros com todas as variações de fontes, geofones e acoplamentos feitos anteriormente, mas de forma simultânea.

\section{Resultados}

Após adquirir os primeiros dados no local de investigação foi feita a parte inicial do processamento com o objetivo de identificar as possíveis reflexões e cotejar o resultado obtido com o sismograma sintético gerado a partir das informações do poço PP1 (Figura 1).

O processamento da sísmica de reflexão rasa é caracterizado pela necessidade de preservar as altas freqüências e as reflexões rasas, mas diferentemente da sísmica profunda, aqui a maioria dos eventos registrados (onda direta, refrações, ground roll e onda aérea) interferem com as reflexões de interesse.

Inicialmente foi aplicado um filtro de frequência passa banda na tentativa de separar os eventos pelo conteúdo espectral. Testou-se diversas bandas de passagem e diferentes rampas para o filtro, mas sem resultados definitivos quanto a melhoria na identificação dos eventos de reflexão ou sensível acréscimo na relação $S / R$.

Obteve-se a seguir o espectro f-k e a partir da análise de seu resultado aplicou-se o filtro $\mathrm{f}-\mathrm{k}$ para eliminar os eventos associados ao ground roll e à onda áerea, tomando-se o cuidado para eliminar também a parte do espectro (na sua parte negativa) associada ao falseamento da onda áerea.

Finalmente aplicando um filtro de freqüência corta - altas limitando a banda em $250 \mathrm{~Hz}$, e um ganho AGC obtivemos uma seção com três evidentes reflexões em $50 \mathrm{~ms}$, relativa ao topo do conglomerado, $75 \mathrm{~ms}$, associada ao topo da argila, e $100 \mathrm{~ms}$, relacionada ao embasamento (Figura 2, Figura 3).

\section{Conclusões}

A análise comparativa de todos os sismogramas dos testes de ruído após o processamento mostrou que os melhores resultados foram obtidos com: (i) geofones de $100 \mathrm{~Hz}$ com ponteira de $0,18 \mathrm{~m}$ e fonte marreta com placa de metal; (ii) geofones de $100 \mathrm{~Hz}$ acoplados através de argila na cobertura asfáltica com fonte marreta aplicada sobre asfalto.

Já as análises dos espectros de amplitude obtidos a partir dos dados do segundo experimento indicam: (1) a ponteira de $0.18 \mathrm{~m}$ não é mais vantajosa do que de 0.14 m (Figura 4); (2) os geofones de $100 \mathrm{~Hz}$ acoplados através de argila no pavimento permitem ter um resultado equivalente a dos geofones acoplados com ponteiras no solo já que a banda de freqüência deles é a mesma, e as perdas da energia não são significativas (Figura 4); (3) para o geofone com a freqüência natural de $100 \mathrm{~Hz}$ observou-se a resposta plana na banda das freqüências de $30 \mathrm{~Hz}$ até $120 \mathrm{~Hz}$ diferentemente de geofone com a freqüência natural de $28 \mathrm{~Hz}$ que no seu espectro mostrou uma acentuada queda de aproximadamente $20 \mathrm{db}$ entre a freqüência predominante $(\sim 20 \mathrm{~Hz})$ e as mais altas freqüências $(>60 \mathrm{~Hz})$, o que explica a pior qualidade do dado adquirido usando geofones com a freqüência natural de $28 \mathrm{~Hz}$ (Figura 4); (4) as fontes marreta e rifle sísmico não apresentaram diferenças na largura da banda, mas mostraram desigualdade na energia introduzida, o que pode explicar pior qualidade do dado adquirido usando rifle sísmico; (5) os resultados obtidos com a marreta aplicada no pavimento e na placa sobre pavimento não apresentam vantagens comparados aos da mareta aplicada na placa sobre solo (Figura 4);

\section{Agradecimentos}

Agradecemos ao CNPq pela concessão da bolsa de estudos; ao IPT pelo empréstimo dos geofones de 100 $\mathrm{Hz}$ e rifle sísmico.

\section{Referências}

Hunter, J.A., Pullan, S.E., Burns, R.A., Gagne, R.M., and Good, R.L., 1984, Shallow seismic reflection mapping of the overburden-bedrock interface with the engineering seismograph - some simple techniques: Geophysics, 49, 1381-1385. 
Jeng, Y., 1995, Shallow seismic investigation of a site with poor reflection quality: Geophysics, 60, 1725-1726.

Prado, R.L., Malagutti Filho, W., and Dourado, J.C., 2001, The use of shalow seismic reflection technique in near surface exploration of urban sites: an evaluation in the city of São Paulo, Brasil: Brazilian Journal of Geophysics, Vol. 19(3), 293-302.

Steeples, D.W., and Miller, R.D., 1990, Seismic reflection methods applied to engineering, environmental and groundwater problems, in Ward, S., Ed.,Geotechnical and
Environmental Geophysics, Volume I: Review and Tutorial: Soc. Expl. Geophys., 1-30

Stockwell, J. W., and Coher, J. K., 1998, The New SU User's Manual: CWP Colorado School Mines, USA, version 2.2

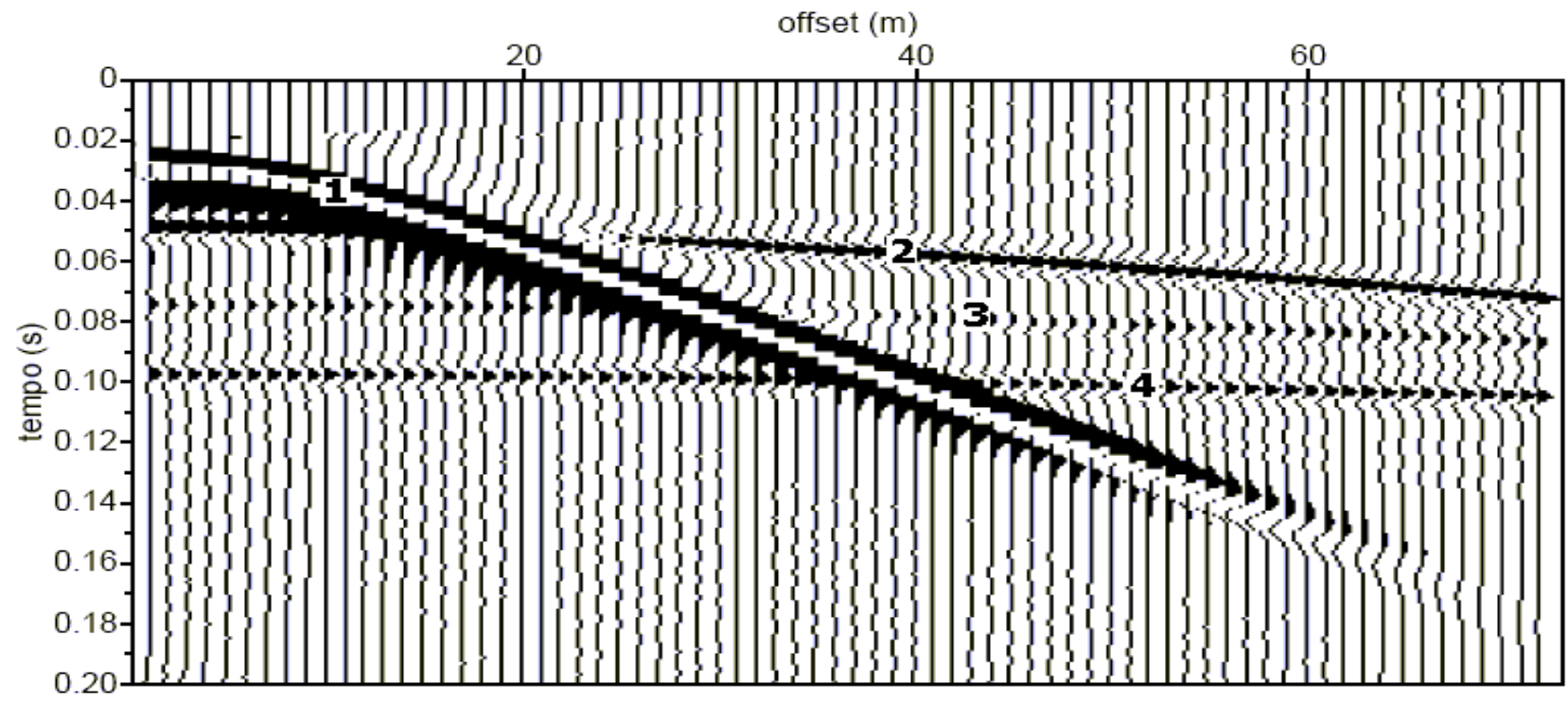

Figura 1 - Sismograma sintético gerado a partir da descrição do poço PP1. Evento 1: interface solo/nível freático, prof. 5 m, $\mathrm{V}_{\text {int }}=0,4 \mathrm{~km} / \mathrm{s}$; evento 2: topo do conglomerado, prof. $25 \mathrm{~m}, \mathrm{~V}_{\mathrm{in}}=1,7 \mathrm{~km} / \mathrm{s}$; evento 3: topo da argila, prof. $52 \mathrm{~m}, \mathrm{~V}_{\text {in }}=2,1 \mathrm{~km} / \mathrm{s}$; evento 4: embasamento, prof. $90 \mathrm{~m}, \mathrm{~V}_{\text {in }}=2,4 \mathrm{~km} / \mathrm{s}$.

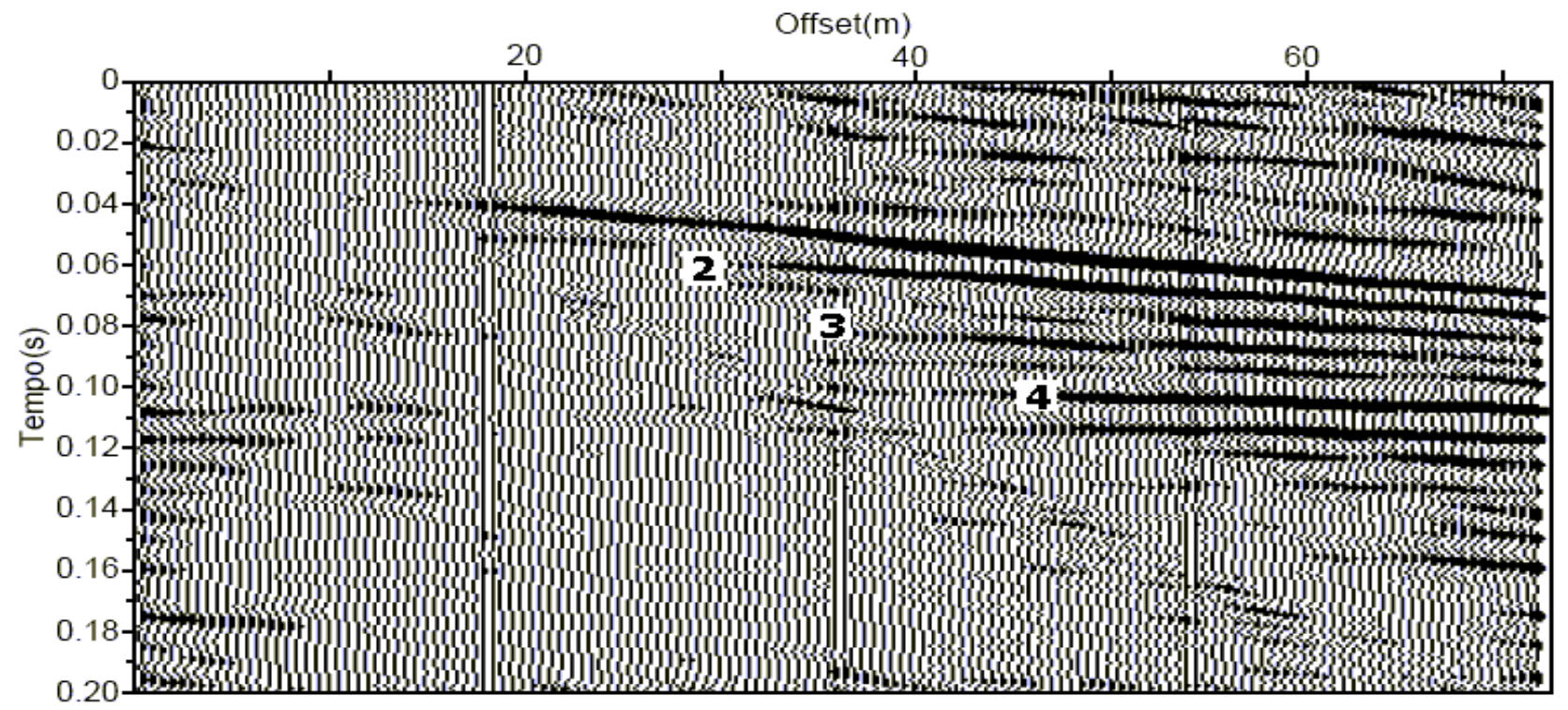

Figura 2 - Registro sísmico obtido com os geofones de $100 \mathrm{~Hz}$ acoplados através das ponteiras de $0.18 \mathrm{~m}$ no solo utilizando a marreta impactada sobre placa de ferro 


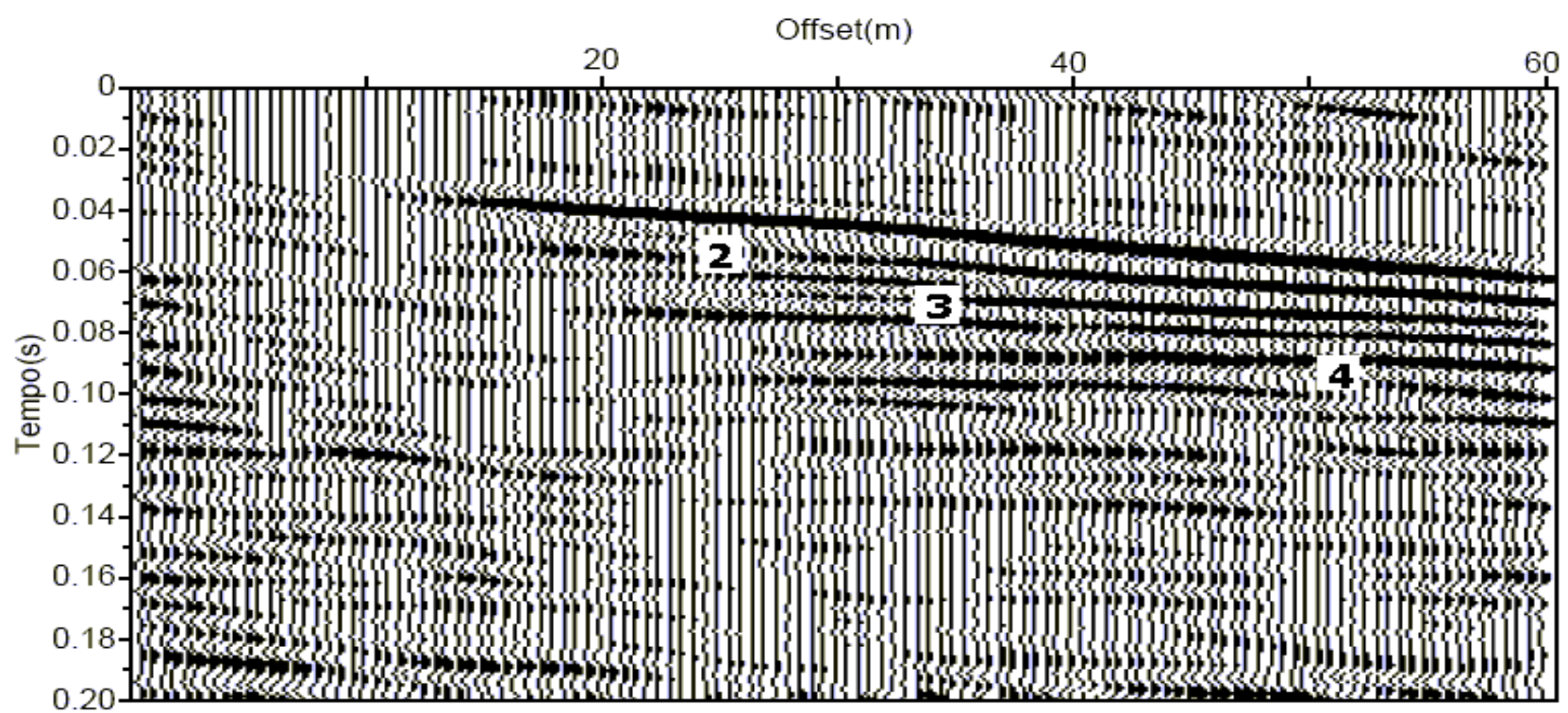

Figura 3 - Registro sísmico obtido com os geofones de $100 \mathrm{~Hz}$ acoplados através de argila no pavimento asfáltico utilizando a marreta impactada sobre a placa de ferro.

a)

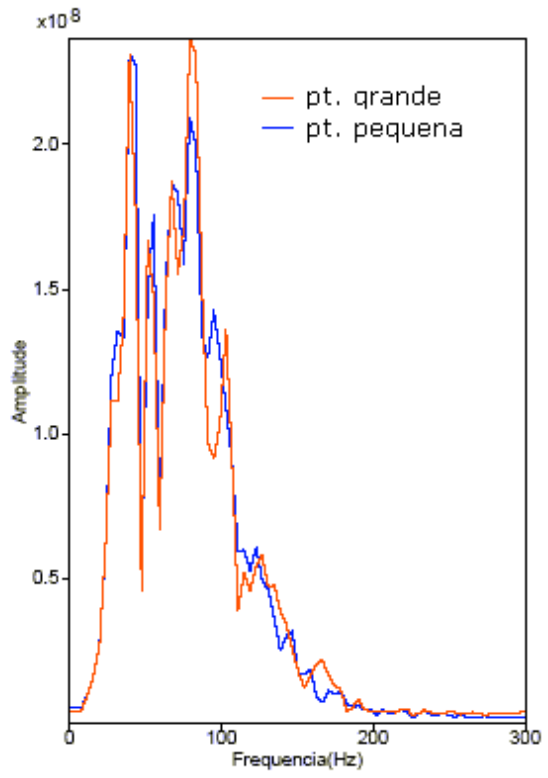

b)

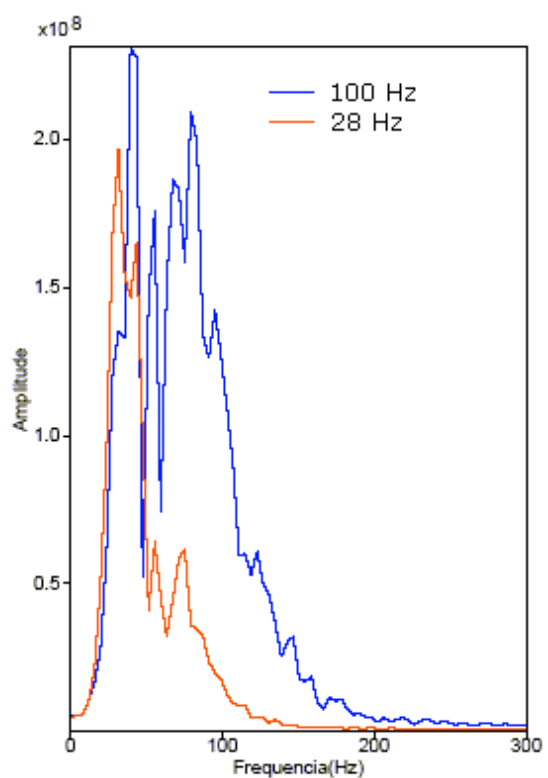

c)

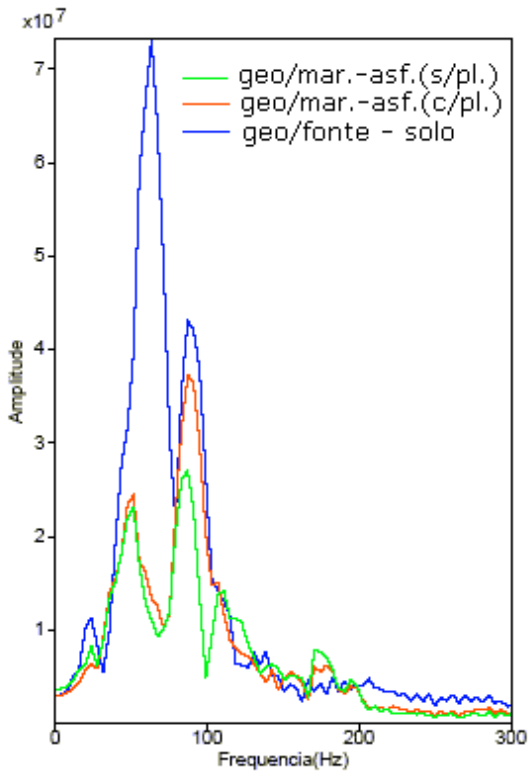

Figura 4 - Espectros dos registros de campo obtidos com diferentes tipos de geofones, fontes e condições de acoplamento: (a) geofones com a ponteira de $0.18 \mathrm{~m}$ e de $0.1 \mathrm{~m}$, fonte marreta ; (b) geofones de $100 \mathrm{~Hz}$ (10 impactos) e $28 \mathrm{~Hz}(1 \mathrm{impacto})$, fonte marreta impactada sobre placa de ferro; (c) geofones de $100 \mathrm{~Hz}$ acoplados com a ponteira de $0.18 \mathrm{~m}$ (azul) com a marreta impactada sobre a placa de ferro, e acoplados através da argila no pavimento com impacto diretamente sobre o pavimento (verde) e sobre placa de ferro (vermelha). 